\title{
ArcheoSciences
}

Revue d'archéométrie

\section{Archéologie précolombienne et analyses scientifiques}

La figurine d'El Angel, une œuvre composite d'orfèvrerie de la culture La Tolita Tumaco (Équateur-Colombie)

Pre-Columbian archaeology and science-based analysis : the figurine of El Angel, a composite goldwork from La Tolita Tumaco culture (Ecuador - Colombia)

Jean-François Bouchard et Maria Filomena Guerra

\section{(2) OpenEdition \\ Journals}

\section{Édition électronique}

URL : https://journals.openedition.org/archeosciences/2315

DOI : $10.4000 /$ archeosciences. 2315

ISBN : 978-2-7535-1598-7

ISSN : $2104-3728$

Éditeur

Presses universitaires de Rennes

\section{Édition imprimée}

Date de publication : 31 décembre 2009

Pagination : 273-279

ISBN : 978-2-7535-1181-1

ISSN : 1960-1360

Référence électronique

Jean-François Bouchard et Maria Filomena Guerra, « Archéologie précolombienne et analyses scientifiques », ArcheoSciences [En ligne], 33 | 2009, mis en ligne le 10 décembre 2012, consulté le 28 janvier 2022. URL : http://journals.openedition.org/archeosciences/2315 ; DOI : https://doi.org/ 10.4000/archeosciences.2315 


\title{
Archéologie précolombienne et analyses scientifiques : la figurine d'El Angel, une œuvre composite d'orfèvrerie de la culture La Tolita Tumaco (Équateur-Colombie)
}

\author{
Pre-Columbian archaeology and science-based analysis : the figurine of El Angel, \\ a composite goldwork from La Tolita Tumaco culture (Ecuador - Colombia)
}

\author{
Jean-François Bouchard* et Maria Filomena GuerRA**
}

\begin{abstract}
Résumé : Une petite figurine en or provenant du site El Angel est associée aux traditions orfevres Tumaco La Tolita, alors qu'elle provient d'une région hors de l'aire culturelle de cette civilisation, à l'extérieur de la frange côtière. L'étude, au moyen de méthodes non-destructives, des techniques de mise en forme, assemblage et décoration utilisées pour sa fabrication montre que la figurine a subi un remontage moderne, mais que la grande majorité de ses parties principales est authentique.

La composition élémentaire des alliages et surtout la présence de fortes teneurs en Fe, Pd et Pt sont typiques des ors utilisés pour l'orfèvrerie La Tolita Tumaco. La figurine provenant du site d'El Angel, en Sierra, serait ainsi un des rares exemples d'objets exportés loin de son aire géoculturelle.
\end{abstract}

\begin{abstract}
A small gold figurine found at the site of El Angel, far from the coast threshold, is usually associated with the Tumaco La Tolita goldsmith traditions, in spite of a location outside the cultural area of this civilisation. The study of the figurine by non-destructive scientific methods, evidencing the different steps of production, such as joining and decoration, shows that the object was remounted. However, the majority of its main parts are genuine. The composition of the alloys and especially the presence of Fe, Pd and Pt are typical of the gold used in the production of La Tolita Tumaco objects. The analytical results attest that the figurine from El Angel, in Sierra, could have been one of the exceptional objects exported far away from its geo-cultural area.
\end{abstract}

Mots-clés : La Tolita Tumaco, analyse, composition, orfèvrerie, El Angel.

Keywords: La Tolita Tumaco, analysis, composition, goldwork, El Angel.

\footnotetext{
* Archéologie des Amériques, UMR 8096 CNRS - Maison René Ginouvès, 21 allée de l’Université, 92023Nanterre Cedex. (jean-francois.bouchard@ mae.u-paris10.fr)

** Laboratoire du Centre de Recherche et de Restauration des Musées de France, UMR171 CNRS, 14, quai François Mitterrand, 75001 Paris, France. (maria.guerra@culture.gouv.fr)
} 


\section{INTRODUCTION}

La métallurgie préhispanique est surtout devenue célèbre à travers les pillages des conquistadors. À peine arrivés dans le Nouveau Monde, ceux-ci ont mis à sac les " trésors » des indigènes. Par la suite, les fouilles clandestines des pillards, visèrent surtout les sépultures et lieux funéraires préhispaniques. Divers pays, comme la Colombie, l'Équateur, le Pérou, la Bolivie, pour ne citer que ceux de la tradition métallurgique andine, ont perdu une grande part de leur orfevrerie, souvent fondue en lingots car les pillards n'y voyaient le plus souvent que des objets bien " monnayables " dans tous les sens du mot. Ces mêmes pillards étaient les premiers sur place pour ouvrir les tombes et les chercheurs n'y avaient accès qu'une fois le pillage terminé. Commençait alors un trafic occulte où les œuvres finissaient par être vendues discrètement et se retrouvaient dans des collections privées et publiques. Ce n'est qu'une très infime partie des œuvres d'orfevrerie de tous les musées publics qui échappent à cette règle générale. Ce rappel permet de comprendre pourquoi les archéologues sont souvent mal informés sur ces œuvres.

Parmi les traditions orfèvres des Andes, l'une des plus anciennes est celle de la civilisation La Tolita Tumaco qui s'est épanouie sur le littoral Pacifique de la Colombie et de l'Équateur (600 av. J.-C.-400 apr. J.-C.). La métallurgie de La Tolita Tumaco a été étudiée par divers auteurs, archéologues et archéomètres (Bergsoe, 1937; Verneau et Rivet, 1912-1922; Scott et Bray, 1994; Plazas, 1998; Bray, 2000; Bouchard et Usselmann, 2003; Scott, 2004).

Un certain nombre de musées possèdent dans leurs collections des œuvres d'orfèvrerie provenant de la région La Tolita Tumaco. Le musée du Quai-Branly, Paris, nous montre ainsi une petite figurine en or, pour laquelle nous avons pu mener une étude particulière. Bien que provenant d'une région hors de l'aire culturelle Tumaco La Tolita (site El Angel, au sud-ouest de Tulcán, dans les environs du volcan Chiles, province du Carchi, Equateur), elle est considérée comme ayant été réalisée par les orfèvres de cette culture (Fig. 1). L'œuvre est composée d'un assemblage de 12 éléments majeurs, façonnés en feuilles d'or martelées, puis mis en forme. Ils sont assemblés par des fils d'aspect métallique et de couleurs différentes. Sur le visage, deux éléments en amande représentant les yeux sont fixés et entourés de fils torsadés. L'absence de données précises dans les enregistrements du musée rendait hypothétique l'attribution culturelle suggérée pour cette œuvre.

L'examen et l'analyse de la figurine du site El Angel au LC2RMF, études peu usuelles en archéologie préhispanique, mettent en évidence les techniques de mise en forme, assemblage et décoration utilisées pour sa fabrication mais aussi la présence de restaurations modernes par addition de certaines parties. L'analyse des alliages a aussi révélé le type d'or utilisé et permis la comparaison avec d'autres productions d'orfevvres de cette aire géoculturelle, de façon à entreprendre une recherche sur la provenance de l'or.

\section{RÉSUltats ANALYTIQUES}

L'examen de la figurine d'El Angel a été réalisé sous loupe binoculaire, par radiographie X et par microscopie électronique à balayage (MEB) avec un Philips XL30 ESEM en mode $S E$ à $20 \mathrm{kV}$. L'analyse élémentaire a été effectuée à l'accélérateur AGLAE au moyen des techniques PIXE et PIGE (particle induced $\mathrm{X}$-ray emission et $\gamma$-ray emission) avec un faisceau de protons de $3 \mathrm{MeV}$ extrait à l'air. La technique PIXE utilise deux détecteurs de $\mathrm{Si}(\mathrm{Li})$, un dédié à la détection des éléments majeurs et l'autre, avec un filtre sélectif de $75 \mu \mathrm{m}$ de cuivre, dédié à la mesure des éléments mineurs et traces; la technique PIGE utilise un détecteur HPGe avec une efficacité de $30 \%$ placé à $45^{\circ}$ avec le faisceau (Guerra et Calligaro, 2004; Guerra, 2004). Le Tableau 1 présente les résultats obtenus pour les régions plates centrales des diverses parties de la figurine d'El Angel. Ces valeurs sont le résultat de deux mesures avec balayage de $200 \mu \mathrm{m}^{2}$ à $500 \mu \mathrm{m}^{2}$, selon les dimensions de la partie analysée.

\section{Les modes de fabrication et les alliages employés}

La radiographie $\mathrm{X}$ pratiquée sur la figurine permet de comprendre le montage des différentes plaques obtenues par martelage et des fils utilisés (Figure 2). La plus grande épaisseur des plaques constituant la jambe et le pied gauches de la figurine semble indiquer un ajout ou un remontage postérieur à la découverte à l'aide de deux éléments modernes copiés sur la jambe droite originale. Cette hypothèse est confortée par l'absence de platine dans l'alliage des plaques suspectes.

Les yeux sont formés chacun de deux éléments : un contour d'œil en forme d'amande, réalisé en fil d'or torsadé et un élément, lui aussi de forme ovale, réalisé en métal de couleur gris-moyen et d'aspect plutôt terne. Dans l'enregistrement d'origine, il est seulement spécifié que l'un des yeux est en platine. Notre analyse a montré que la plaque blanchâtre de l'œil droit a bien été fabriquée avec un alliage or-platine, obtenu par frittage (en anglais sintering, p. ex. Scott et Bray, 1994). Par contre, l'analyse de la plaque noirâtre de l'œil gauche montre qu'elle est composée d'argent et de cuivre, ce serait un ajout postérieur effectué lors du remontage de la jambe gauche. 

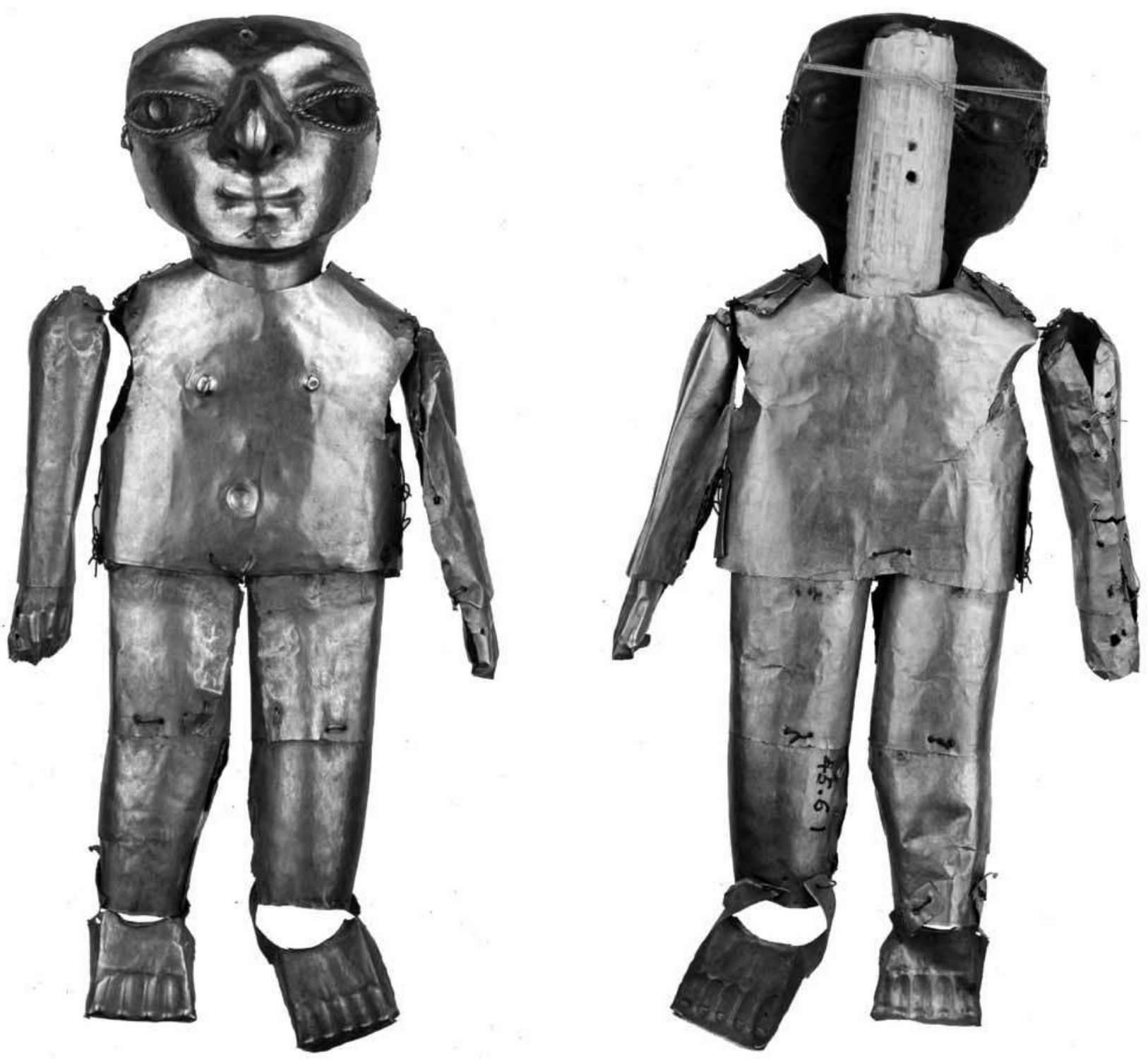

Figure 1 (a) et (b) : La figurine composite d'El Angel (or, argent, platine). Dimensions : hauteur $13 \mathrm{~cm}$; largeur $6 \mathrm{~cm}$ (C) C2RMF, D. Bagault).

Figure 1 (a) et (b): The composite figurine found at the site of El Angel (gold, platinum, silver). Dimensions: $13 \mathrm{~cm}$ high; $6 \mathrm{~cm} \mathrm{large} \mathrm{(C)} \mathrm{C2RMF,}$ D. Bagault).

Ces résultats sont en accord avec les conclusions de d'Harcourt publiées en 1948. Par ailleurs, la composition de l'œil droit se situe dans la région chimique définie par les teneurs en platine, or et argent obtenues par Bergsoe (1937) et par Scott and Bray (1994) pour des objets attribués à La Tolita. La teneur en fer de l'alliage est de 3,3\% ce qui se situe dans les valeurs types avancées par Scott (1998) pour les grains de platine natif d'Amérique du Sud, typiquement de 1 à $4 \%$. Il semble donc que l'œil droit ait une composition conforme à celle qu'on est en droit d'attendre pour un objet Tumaco La Tolita.
L'observation détaillée des yeux sous loupe binoculaire met en évidence les deux types de fabrication des fils torsadés formant le contour de l'œil. Malgré l'utilisation de fils lisses pleins dans les deux cas, l'œil en or-platine est entouré d'un fil façonné par martelage, dont l'alliage possède en moyenne environ $2 \%$ de platine, alors que l'œil en argent-cuivre est entouré d'un fil fabriqué par tréfilage, avec un alliage sans platine, et il est donc nécessairement moderne.

Les différents éléments constituant la figurine sont assemblés à l'aide de fils. Dans certains cas, l'assemblage est assez discret, comme pour la jonction de parties des jambes, mais, 


\begin{tabular}{|c|c|c|c|c|c|c|c|c|c|c|c|c|}
\hline & & $\mathrm{Au} \%$ & $\mathrm{Ag} \%$ & $\mathrm{Cu} \%$ & $\mathrm{Zn} \%$ & Pt \% & $\begin{array}{c}\mathrm{Ru} \\
\mathrm{ppm}\end{array}$ & $\begin{array}{c}\mathrm{Rh} \\
\mathrm{ppm}\end{array}$ & $\begin{array}{c}\mathrm{Pd} \\
\mathrm{ppm}\end{array}$ & $\begin{array}{c}\text { Sn } \\
\text { ppm }\end{array}$ & $\begin{array}{c}\mathrm{Sb} \\
\mathrm{ppm}\end{array}$ & $\begin{array}{c}\mathrm{Fe} \\
\mathrm{ppm}\end{array}$ \\
\hline \multirow{2}{*}{ œil droit } & plaque & 54,6 & 8,6 & 1,0 & & 32,3 & 810 & 6010 & 3175 & & & 33000 \\
\hline & \begin{tabular}{|l|} 
filigrane \\
\end{tabular} & 82,7 & 12,7 & 2,7 & & 1,8 & 40 & 90 & 200 & & & 385 \\
\hline \multirow{2}{*}{ œil gauche } & plaque & 0,4 & 49,5 & 47,1 & & & & & & 1650 & 170 & 200 \\
\hline & filigrane & 76,9 & 17,3 & 4,8 & & & & & & 320 & & 650 \\
\hline \multirow{3}{*}{ jambe gauche } & pied & 76,5 & 17,2 & 5,0 & & & & & & & & 40 \\
\hline & jambe & 76,3 & 17,3 & 5,5 & & & & & & 390 & 145 & 730 \\
\hline & cuisse & 79,4 & 10,4 & 7,2 & & 2,8 & 60 & 380 & 210 & 85 & 30 & 35 \\
\hline \multirow{3}{*}{ jambe droite } & pied & 78,0 & 10,8 & 8,3 & & 2,8 & 140 & 460 & 260 & & & 50 \\
\hline & jambe & 77,3 & 11,8 & 8,5 & & 2,3 & 190 & 430 & 300 & & & 800 \\
\hline & cuisse & 78,1 & 10,9 & 8,2 & & 2,7 & 40 & 340 & 320 & & & 45 \\
\hline \multirow{2}{*}{ torse } & avant & 77,6 & 10,5 & 8,3 & & 2,0 & & 330 & 325 & 170 & 50 & 45 \\
\hline & arrière & 78,2 & 12,9 & 5,8 & & 3,0 & & 370 & 390 & & & 550 \\
\hline \multirow{2}{*}{ bras gauche } & bras & 78,6 & 9,0 & 9,5 & & 2,6 & 40 & 220 & 225 & & & 1220 \\
\hline & \begin{tabular}{|l|} 
main \\
\end{tabular} & 78,9 & 9,9 & 8,6 & & 2,4 & 90 & 210 & 270 & & & 520 \\
\hline \multirow{2}{*}{ bras droit } & bras & 79,7 & 10,7 & 7,9 & & 1,6 & 28 & 115 & 185 & & & 540 \\
\hline & main & 78,1 & 10,6 & 8,6 & & 2,7 & & 215 & 290 & & & 490 \\
\hline \multirow{2}{*}{ tête } & nez & 71,1 & 12,0 & 6,4 & & 9,7 & 826 & 3230 & 725 & & & 6735 \\
\hline & menton & 77,7 & 10,7 & 8,8 & & 2,6 & & 270 & 355 & & & 485 \\
\hline \multirow{4}{*}{$\begin{array}{l}\text { fils } \\
\text { d'assemblage }\end{array}$} & $\begin{array}{l}\text { main / bras } \\
\text { gauche }\end{array}$ & 74,1 & 20,4 & 4,4 & & & & & & 162 & 168 & 795 \\
\hline & jambe gauche & & & 61,9 & 36,2 & & & & & & & 645 \\
\hline & $\begin{array}{l}\text { bras gauche / } \\
\text { torse }\end{array}$ & 75,4 & 18,0 & 5,5 & & & & & & & & 770 \\
\hline & bras droit / torse & & & 62,8 & 34,4 & & & & & 104 & 1014 & 610 \\
\hline
\end{tabular}

Tableau 1 : Résultats obtenus par PIXE pour les différentes parties de la figurine d'El Angel.

Table 1: Compositional results on all the analysed parts of the El Angel figurine by PIXE. ailleurs, il peut être plutôt grossier et, même, fait au moyen de fils tortillés qui dépassent à l'extérieur de la figurine, en particulier à la jonction des bras et des épaules (figure 3b). D'après Scott (Scott, 2004), les fils utilisés par les orfèvres de la civilisation La Tolita Tumaco sont typiquement façonnés par martelage quand le but est de produire des attaches de parties de fabrication indépendante. En ce qui concerne les fils de décoration, ils sont généralement pleins-tordus et martelés, mais aussi parfois tubulaires-tordus (ou en très fine tôle d'or roulée sur elle-même).

Les fils de la figurine d'El Angel peuvent être lisses pleins, d'aspect noirâtre (figure 3a) ou jaune, et aussi tubulaires de couleur jaune. Leur analyse montre que les fils jaunes sont fabriqués à partir d'alliages d'or et les fils noirâtres sont en laiton. Ces caractéristiques suggèrent l'hypothèse d'un remontage des parties principales, après la découverte de l'œuvre avec un matériau d'origine non archéologique, au moins en partie. Le fait que le dernier type d'alliage soit inconnu à l'époque préhispanique confirme bien l'hypothèse d'un remontage moderne.

\section{Hypothèses de provenance de l'or}

La présence d'éléments du groupe du platine dans les ors de la région du Nord-Ouest de l'Amérique du Sud, ainsi que la composition du platine natif, sont connus depuis de longues années, grâce aux analyses effectuées au XIX ${ }^{\mathrm{e}}$ siècle par Berzelius pour la Colombie (Berzelius, 1928) et par Wolf pour Esmeraldas (Wolf, 1912).

L'analyse des différents éléments supposés originaux de la figurine d'El Angel montre qu'ils ont été fabriqués à partir d'un alliage dont la composition moyenne est de $77,7 \pm 2,1 \%$ d'or, $10,8 \pm 1,0 \%$ d'argent, $8,0 \pm 1,0 \%$ de cuivre et $2,5 \pm 0,4 \%$ de platine (remarquons la présence d'inclusions de platinoïdes, identifiées au MEB-EDS et constituées d'Ir, Pt et Os). La comparaison de cet alliage avec la composition des objets publiés par Bergsoe, 1937; Scott et Bouchard, 1988 ; Rovira, 1994; Scott et Bray, 1994; Estevez, 1998 ; Barrandon et al., 2004 et Valdez et al., 2005, montre que la figurine s'approche des réalisations des orfèvres de la civilisation La Tolita Tumaco. Il s'avère donc que cet alliage est bien différent de celui utilisé dans la fabrication des parties que nous supposons être des ajouts modernes. En effet, les fils d'assemblage, le fil de l'œil gauche et les plaques de la jambe et pied gauches ont une composition moyenne de : $75,8 \pm 1,1 \%$ d'or, $18,1 \pm 1,3$ d'argent et $5,1 \pm 0,5 \%$ de cuivre; le platine ne pourrait être présent qu'à des quantités inférieures aux limites de détection. La présence de platinoïdes dans les parties jugées antiques semble ainsi suffisante pour indiquer que la plupart des éléments constituant la figurine paraissent bien originaux.

De façon à vérifier l'attribution de l'or utilisé dans la fabrication des parties originales aux gisements alluvionnaires de la côte, nous avons comparé nos résultats pour les éléments 


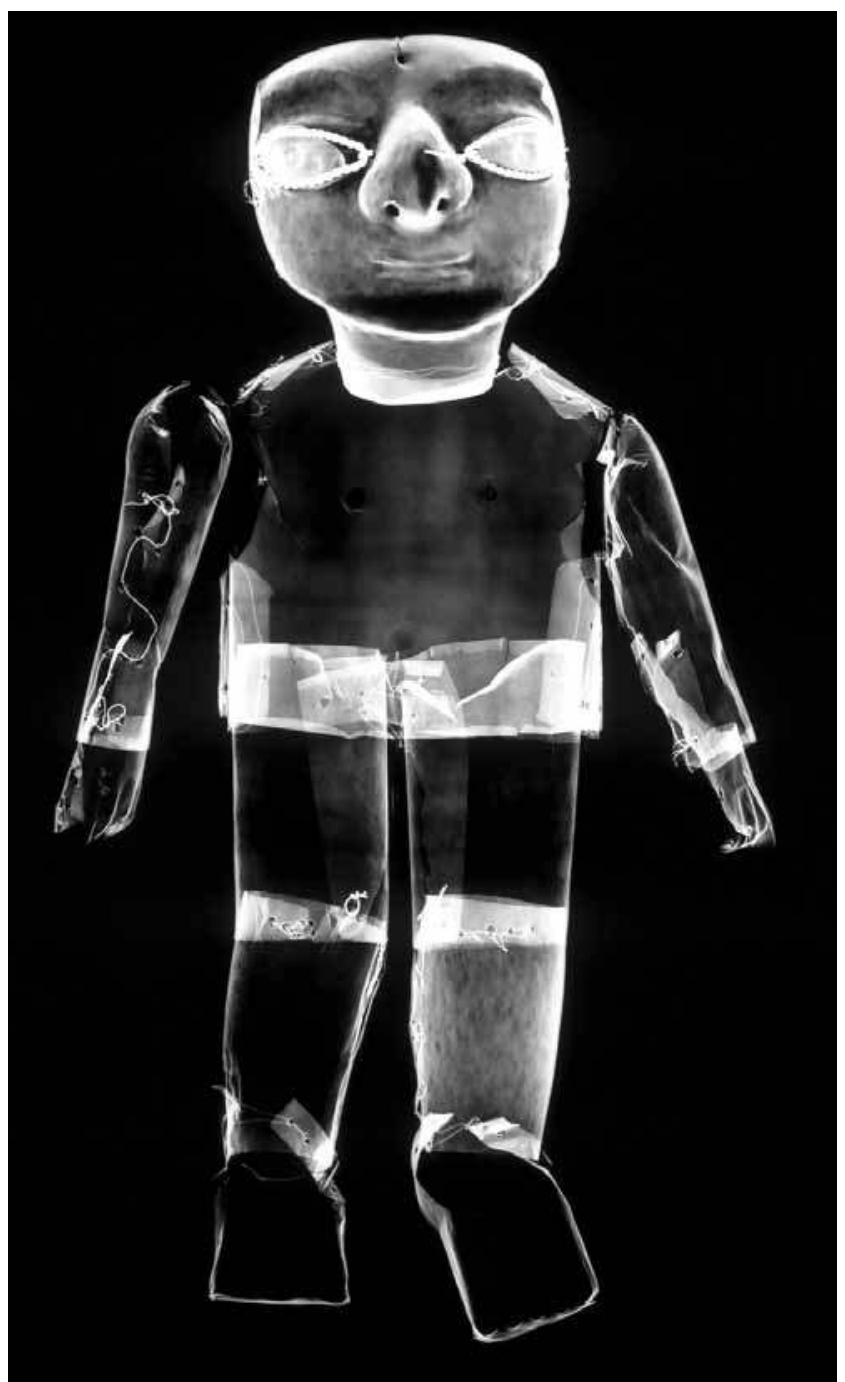

Figure 2 : (Voir planche couleur) Radiographie aux rayons X de la figurine d'El Angel (@ C2RMF, T. Borel).

Figure 2: (See colour plate) X-radiography of the figurine from $\mathrm{El}$ Angel (C) C2RMF, T. Borel).

traces avec les compositions de divers objets de La TolitaTumaco et d'Esmeraldas publiés par Barrandon et al., 2004 et par Valdez et al., 2005. Parmi les éléments traces les plus significatifs nous avons sélectionné le palladium et le platine. Sur la Figure 4 ces deux éléments sont corrélés (corrélation typique des gisements alluvionnaires) pour les objets de La Tolita, des objets fabriqués par les civilisations côtières, ainsi que pour la figurine d'El Angel.

Les objets provenant des régions de la Sierra possèdent des teneurs plus basses et plus dispersées en palladium et platine. Ceci peut être expliqué par l'utilisation des gisements auriferes de type primaire ainsi que par un possible mélange d'or alluvionnaire avec de l'or filonien.
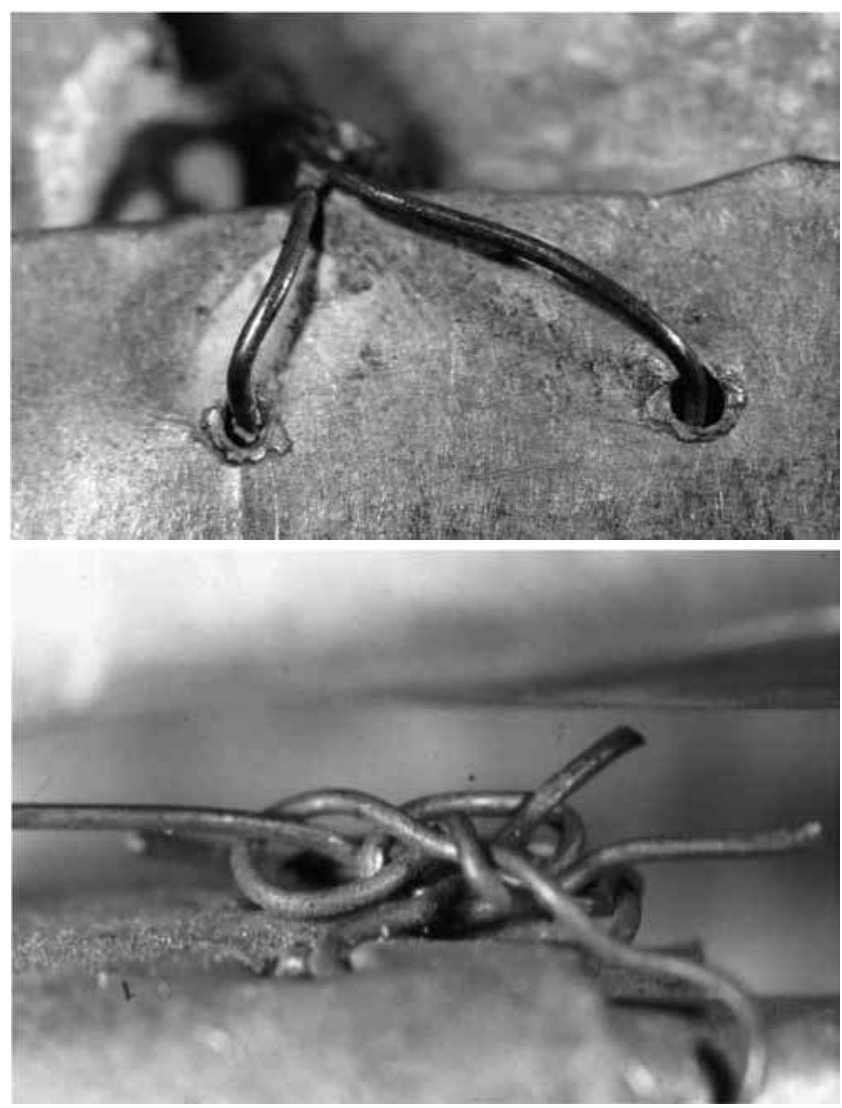

Figure 3 : (Voir planche couleur) Images sous loupe binoculaire des fils : (a) noirâtres et (b) tortillés (C) C2RMF, D. Bagault).

Figure 3: (See colour plate) Low magnification micrographs under the binocular of the wires: (a) blackish and (b) wriggled (C) C2RMF, D. Bagault).

L'or de la statuette d'El Angel s'approche bien de l'or des alliages utilisés par les différents groupes d'orfèvres qui ont travaillé le métal à La Tolita. Non seulement la statuette d'El Angel présente des analogies stylistiques de cette région côtière, comme il ressort de l'étude morphologique et technologique, mais surtout les parties originales de cette œuvre sont très proches en composition des divers produits d'orfèvrerie réalisés à partir des matières premières obtenues dans les mêmes types de gisements auriferes.

\section{Conclusion}

Les analyses et examens menés sur la figurine d'El Angel suggèrent bien qu'elle a fait l'objet de diverses interventions modernes, en particulier un remontage maladroit et l'ajout de parties non préhispaniques. Sont suspects : l'œil gauche (en alliage d'argent-cuivre) et le fil du contour de cet œil gauche, la jambe gauche et, sans doute, le pied gauche, la 


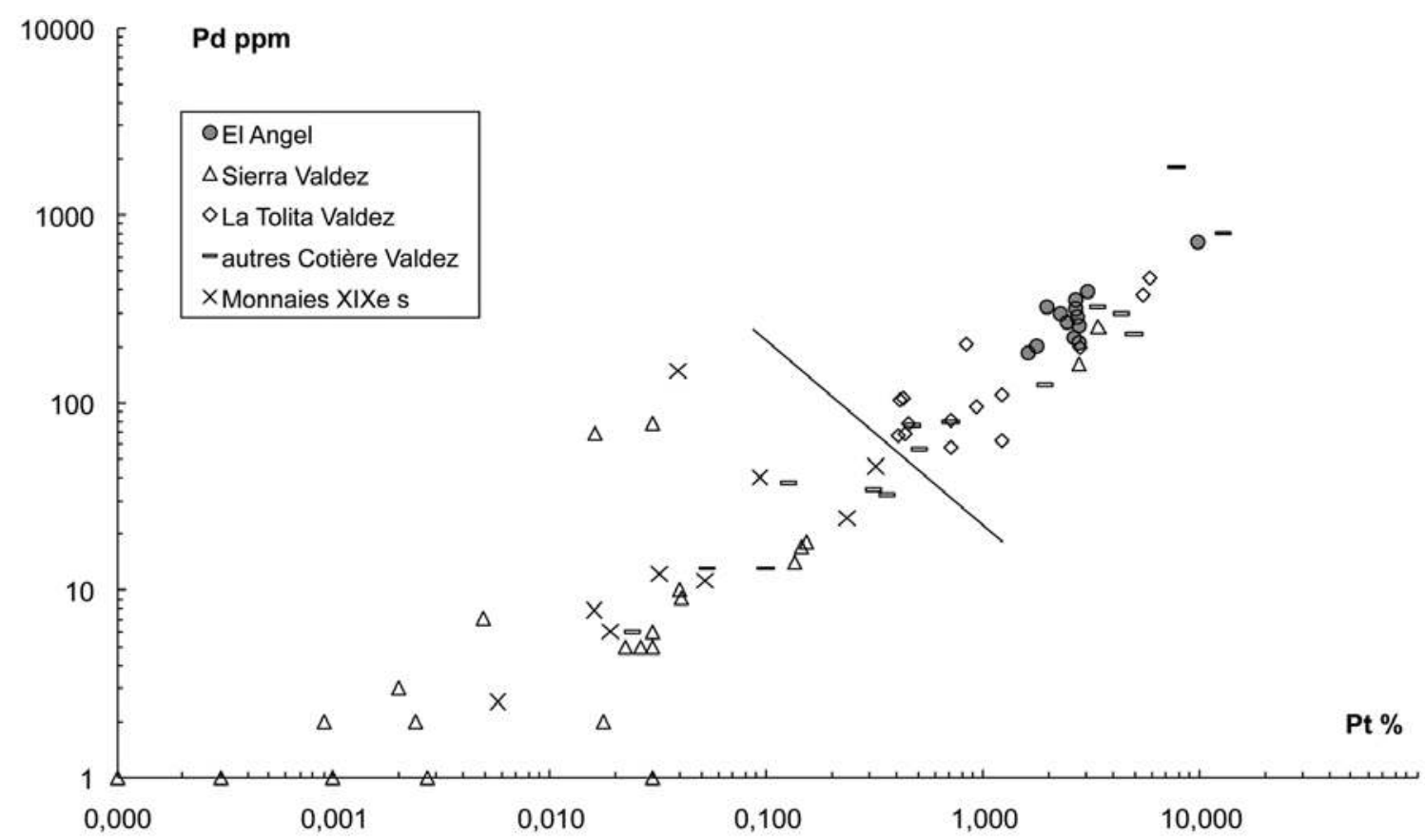

Figure 4 : Représentation des concentrations en Pd et en Pt (en ppm) pour la figurine d'El Angel et pour divers objets de La Tolita Tumaco et d'Esmeraldas publiés par Barrandon et al., 2004 et par Valdez et al., 2005 et des monnaies sud américaines (Guerra, $2004 \mathrm{~b})$.

Figure 4: Pd and Pt contents (in ppm) for the figurine from El Angel and for objects from La Tolita Tumaco and Esmeraldas published by Barrandon et al., 2004 and Valdez et al., 2005 and South America coins (Guerra, 2004b).

plupart des fils d'assemblage (voire tous). Nous pouvons aussi remettre en question l'articulation et la mobilité des parties de la figurine : elles n'étaient qu'une simple conséquence de la restauration moderne. À l'origine, elle avait certainement été assemblée fermement et il n'était pas possible de lui donner diverses postures en déplaçant ses membres ou sa tête. Il n'y avait donc pas d'articulation mais un assemblage. Cela est d'ailleurs plus conforme au montage d'autres figurines La Tolita Tumaco connues. Pour tenter d'évaluer l'époque de l'intervention moderne, on notera que les parties suspectes, de par leurs compositions élémentaires, correspondent à une métallurgie européenne.

L'analyse a donc bien confirmé des soupçons sur quelques éléments de la figurine d'El Angel, mais, la grande majorité des parties principales semblent authentiques et typiques par leur composition élémentaire de cette orfèvrerie La Tolita Tumaco. La figurine, si elle provient bien du site d'El Angel, en Sierra, n'a donc pas été trouvée dans la frange côtière correspondant au territoire La Tolita Tumaco. Pourtant par sa composition, l'or utilisé dans la fabrication de la figurine possède les éléments caractéristiques espérés pour un travail d'orfèvrerie de cette zone culturelle. Cette figurine serait ainsi un des rares exemples d'objets exportés loin de son aire géoculturelle.
Dans l'état actuel de nos connaissances, il reste néanmoins inexplicable qu'elle soit ainsi parvenue dans les hautes terres.

\section{Remerciements}

Nous remercions Christiane Naffah, alors chargée du Chantier des Collections du Musée du Quai Branly, MarieFrance Fauvet-Berthelot, responsable des collections Amérique du Musée de l'Homme, et Thierry Borel, Dominique Bagault et l'équipe AGLAE du C2RMF, qui ont rendu possible l'étude.

\section{Bibliographie}

Barrandon, J.-N., EstéVez, P. et ValdeZ, F., 2004. Identificación mineralogica de las Fuentes del oro precolombino utilizado en la metalurgia prehispanica del Ecuador. Anejos de AEspa XXXII, 405-416.

Berzelius, J.-J., 1838. Traité de chimie, Tome $1^{\mathrm{er}}$, Société Typographique Belge, A. Wahle et C., Bruxelles.

Bergsoe, P., 1937. The Metallurgy and Technology of Gold and Platinum among the precolumbian Indians. (Translated by F. C. Reynolds). Ingeniorvidenskabelige Skrifter $\mathrm{n}^{\circ}$ A44, 
Naturvidenskabelige Samfund i Kommission hos GEC Gad, Copenhagen.

Bouchard, J.-F. et Usselmann, P., 2003. Trois millénaires de civilisation entre Colombie et Équateur, La région Tumaco La Tolita, Paris, CNRS.

BRAY, W., 2000. Malagana and the goldworking tradition of Southwest Colombia. Colombian Gold : technology, style and iconography, The Trustees of The British Museum, 94-111.

D'Harcourt, R., 1948. Archéologie d'Emeraldas et de Manabí, Equateur, Journal de la Société des Américanistes 37, 319-325.

Estevez de Romero, P., 1998. Platino en el Ecuador precolombino, Boletín Museo del Oro 44-45: 159-182.

GuERrA, M. F., 2004a. Fingerprinting ancient gold with proton beams of different energy, Nucl. Instrum. and Methods B 226, 185-198.

Guerra, M. F., 2004b. The circulation of South American precious metals in Brazil in the end of the $17^{\text {th }}$ century, Journal of Archaeological Sciences, 31: 1225-1236.

Guerra, M. F. et Calligaro, T., 2004. Gold traces to trace gold. Journal of Archaeological Sciences, 31: 1199-1208.

Plazas, C., 1998. Cronología de la metalurgia colombiana, Boletín Museo del Oro 44-45: 3-78.

Rovira, S., 1994. Pre-hispanic goldwork from the Museo de América, Madrid : a new set of analysis. Archaeometry of Pre-Columbian
Sites and Artefacts, D. A Scott and P. Meyers ed., The Getty Conservation Institute, Los Angeles: 323-350.

Scott, D. A., 2004. Gold and platinum metallurgy of La Tolita : a metalworking centre of the Pacific lowlands of Ecuador, Anejos de AespA XXXII, 63-82.

Scott, D. et Bray, W., 1980. Ancient Platinum Technology in South America, Platinum Metals Review 24: 147-157.

Scotт, D. A., 1998. Technical examination of ancient South American metals: some examples from Colombia, Peru and Argentina, Boletin Museo del Oro 44-45: 79-115

ScotT, D. et Bouchard, J.-F., 1988. Orfebreria prehispánica de la llanuras del pacifico de Ecuador y Colombia, Boletín Museo del Oro 22: 2-16.

Scott, D. A. et Bray, W., 1994. Pre-Hispanic platinum alloys: their composition and use in Ecuador and Colombia. Archaeometry of Pre-Columbian Sites and Artefacts, D. A. Scott and P. Meyers ed., The Getty Conservation Institute, Los Angeles: 285-322.

Valdez, F., Gratuze, B., Yépez, A. et Hurtado, J., 2005. Evidencia temprana de metalurgia en la Costa Pacifica ecuatorial, Boletín Museo del Oro 53: 1-9.

Verneau, R. et Rivet, P., 1912-1922. Ethnographie de l'ancien Equateur, (2 vol.) Ministère de l'instruction publique, Paris.

Wolf, T. H., 1892. Geografía y geología del Ecuador, Casa de la Cultura Ecuatoriana. $2^{\mathrm{e}}$ ed. 1975: 363-366. 
